\title{
Systematic studies on Asteraceae in the northern region of Bangladesh
}

\author{
A. H. M. Mahbubur Rahman \\ Department of Botany, University of Rajshahi, Rajshahi-6205, Bangladesh
}

Email address:

ahmmahbubur_rahman@yahoo.com

To cite this article:

A. H. M. Mahbubur Rahman. Systematic Studies on Asteraceae in the Northern Region of Bangladesh. American Journal of Life Sciences. Vol. 1, No. 4, 2013, pp. 155-164. doi: 10.11648/j.ajls.20130104.13

\begin{abstract}
Systematic investigation on the family Asteraceae (Compositae) growing throughout in the northern region of Bangladesh was carried out. A total of 36 species under 29 genera of the family Asteraceae were collected and identified. A complete taxonomic account of each species has been given along with their current name, local name, brief description, phenology, basic chromosome number, anatomy and voucher number. Among the recorded species, both anomocytic and anisocytic stomata were found in 21 species; anomocytic stomata in 10 species; anisocytic stomata in 3 species; anomocytic and paracytic stomata were found in 1 species; anomocytic, anisocytic and paracytic stomata were found in 1 species. Out of the 36 species, stomata were found to be present both on the upper and lower surface in 33 species and only on lower surface in 3 species. Three types of trichomes, i.e. non-glandular multicellular, non-glandular unicellular and glandular multicellular trichomes were recorded. Non-glandular multicellular trichome was found in 25 species, nonglandular unicellular in 1 species and glandular multicellular trichome was found in 1 species. Trichomes were absent in rest of the species. Finally, a new system of classification of the family Asteraceae has been proposed on the basis of morphological, anatomical, cytological and floral characters.
\end{abstract}

Keywords: Systematic Investigation, Asteraceae, Northern Region, Bangladesh

\section{Introduction}

The family Asteraceae or Compositae (known as the aster, daisy, or sunflower family) is the largest family of flowering plants, in terms of number of species. The name 'Asteraceae', is derived from the type genus Aster, while 'Compositae', an older but still valid name, means Compositae and refers to the characteristic inflorescence, a special type of pseudanthium found in only a few other angiosperm families [18].

According to the Royal Botanical Gardens of Kew the family comprises more than 1600 genera and 23,000 species. The largest genera are Senecio (1500 species), Vernonia (1000 species), Cousinia (600 species), Centaurea (600 species). The circumscription of the genera is often problematic and some of these have been frequently divided into minor subgroups [10].

Asteraceae (Compositae) is an advanced and botanically highly specialized family of dicotyledons representing mainly herbaceous plants. They are widely distributed in the tropics and warm temperate regions of South, SouthEast and East-Asia, Africa including Madagascar and central South America. The family is represented by about 950 genera and 20,000 species over the globe [20].

Compositae is the largest family of flowering plants. According to [19] Compositae is a very large family with 900 genera and 13,000 species. Compositae is the largest family of angiosperms and the family is represented by about 1000 genera and 20,000 species [14]. They are widely distributed in warm temperate region and Arctic region or Alpine region. The family is represented by about 1100 genera and 25,000 species in the world [11].

The Compositae is the largest family of vascular plants, and the genera are estimated to number about 950 genera and the species probably 20,000. They are distributed over most of the earth and in almost all habitats. The greater proportion are herbaceous, although about 2 percent are trees or shrubs. The family Asteraceae is known as Aster, Daisy or Sunflower family. Some of the genera of this family Aster, Helianthus, Cosmos, Dahlia, Chrysanthemum, Tagetes etc. are ornamentals and most of them have medicinal values. Many species of this family are the sources of medicine. Some are widely cultivated in the field for vegetable and food [13]. 


\section{Materials and Methods}

Systematic investigation on the family Asteraceae growing throughout in the northern region of Bangladesh was carried out from January 2005 to June 2008. The study area of northern region includes six districts and these are Rajshahi (including three thanas Boalia, Godagari, Tanore), Nawabgonj (including three thanas Sadar, Nachole, Sibgonj), Naogaon (including two thanas Atrai, Raninagar), Joypurhat (including two thanas Akkelpur, Khetlal), Bogra (including two thanas Adamdighi, Dupchachia) and Natore (including three thanas Sadar, Singra, Lalpur). A total of 36 species under 29 genera of the family Asteraceae were collected and identified.

A survey on the determination of the location of different species was made and a list was prepared to be acquainted with the Asteraceae available in the selected area. All the species were noted and time to time the areas were visited to see when they flowered. For the morphological study, different types of species were examined again and again in order to see if there was any variation or not. They were collected at flowering stages and herbarium specimens were prepared as vouchers. In this practice standard method was followed. In this regard different types of plant species were collected from different habitats. All the collected plant specimens were kept in the Herbarium, Department of Botany, University of Rajshahi, Bangladesh.

The collected specimens were identified studying related taxonomic books and booklets from the library of Rajshahi University. The major collected materials were identified and described up to species with the help of [6], [17], [3] and [12]. In some cases [5], [1], [2] and [7] were consulted. For the current name and up to date nomenclature [8], [9], [15] and [16] were also consulted.

\section{Results and Discussion}

Systematic investigation on the family Asteraceae (Compositae) growing throughout in the northern region of Bangladesh was carried out. A total of 36 species under 29 genera of the family Asteraceae were collected and identified. Most of the wild species grow in all districts in the study area. Some of the species which was sometimes cultivated and sometimes wild grow in particular area. Out of 36 species 13 species were distributed all over the study area, these were Ageratum conyzoides, Blumea lacera, Dahlia hybrida, Eclipta alba, Enhydra fluctuans, Gnaphalium indicum, Helianthus annuus, Tagetes patula, Tridax procumbens, Vernonia patula, Mikania cordata, Wedelia chinensis and Xanthium indicum.

Out of the total number of species Ageratum conyzoides Linn, Blumea lacera (Burm. f.) DC, Eclipta alba (L.) Hassk, Tridax procumbens Linn, Vernonia patula (Dryand) Merril and Xanthium indicum Linn. Was densely populated and were found in sandy roadside areas of Rajshahi, Naogaon, Nawabgonj, Natore, Bogra and Joypurhat. Among the 36 species, 35 was herbaceous. Mikania cordata (Burm. f.) Robin. was the only climbing species all over the study areas. Among the species studied, Blumea laciniata (Roxb.) DC. and Wedelia trilobata (L.) A.S. Hitchc. have been reported here for the first time from northern region, and Parthenium hysterophorus Linn. was a new record from Bangladesh.

The common species found in the six districts were Ageratum conyzoides, Blumea lacera, Blumea laciniata, Callistephus chinensis, Dahlia variabilis, Eclipta alba, Enhydra fluctuans, Grangea maderaspatana, Helianthus annuus, Launaea aspleniifolia, Mikania cordata, Perthenium hysterophorus, Sonchus arvensis, Spilanthes paniculata, Synedrella nodiflora, Tagetes patula, Tridax procumbens, Vernonia patula, Wedelia chinensis and Xanthium indicum.

By applying survey, plant collection and identification methods, both of anatomical and cytological information were accumulated. The check listed information about the plant materials collected from the study area were described below.

\subsection{Ageratum Conyzoides Linn}

Local name: Oochunti, Fulkuri

Brief description: Annual herbs, 30-90 cm high; stem erect, branched, more or less hairy. Heads small, in dense terminal corymbos; flowers pale blue or white, Pappus of 5-scales, aristate, dilated at the base, serrulate, about equalling the corolla. Achenes 2-2.5 mm long, sharply angled, black.

Phenology: December to May

Basic chromosome number: $\mathrm{X}=10,17,19$ [4]

Anatomy: Stomata:Upper surface: Anomocytic; Lower surface:Anomocytic and anisocytic

Tricome: Non-glandular; multicellular

Voucher number: M.R. 01 Motihar, Rajshahi 06-04-05; M.R.36 Sadar, Natore 07-05-05; M.R. 61 Sadar, Nawabgonj 01-06-05; M.R. 86 Adamdighi, Bogra 02-07-05; M.R. 111 Atrai, Naogaon 04-08-05; M.R. 136 Akkelpur, Joypurhat 08-09-05.

\subsection{Blumea Lacera (Burm f) DC}

Local name: Kukurmuta, kukursunga

Brief description: Annual, with a strong odour of turpentine; stem erect, 0.3-0.9 m high, ash-coloured. Heads 6-8 mm diam., numerous in short axillary cymes and terminal spiciform panicles; flowers yellow. Corolla lobes of hermaphrodite flowers nearly glabrous. Receptacle glabrous. Pappus white. Achenes not ribbed, small, oblong, sub-4-gonous.

Phennology: January to May

Basic chromosome number: $\mathrm{X}=5,7,8,9$ [4]

Anatomy: Stomata: Upper surface: Anisocytic; Lower surface:Anomocytic. Trichome: Non-glandular; multicellular.

Voucher number: M.R. 02 Motihar, Rajshahi 08-04-05; M.R.37 Sadar, Natore 08-05-05; M.R. 62 Nachole, Nawab- 
gonj 02-06-05; M.R. 87 Adamdighi, Bogra 03-07-05; M.R. 112 Atrai, Naogaon 05-08-05; M.R. 137 Akkelpur, Joypurhat 10-09-05.

\subsection{Blumea Laciniata (Roxb) DC}

Local name: Kukurmuta, kukursunga

Brief description: An erect, usually stout, pubescent or laxly tomentose, stem branched, leaves usually large membranous runcinate lyrate or subpinnatifid and toothed, heads usually in broad open corymbs, involucral bracts lanceolate acuminate villous, recept, pubescent corolla yellow. Achenes ribbed silky.

Phennology: December to May

Basic chromosome number: $\mathrm{X}=5,7,8,9$ [4]

Anatomy: Stomata: Upper surface: Anomocytic; Lower surface:Anomocytic. Trichome: Non-glandular; multicellular.

Voucher number: M.R. 10 Boalia, Rajshahi 25-04-05; M.R.45 Sadar, Natore 15-05-05; M.R. 72 Sadar, Nawabgonj 05-06-05; M.R. 90 Dupchachia, Bogra 05-0705; M.R. 116 Raninagar, Naogaon 07-08-05; M.R. 140 Khetlal, Joypurhat 11-09-05.

\subsection{Callistephus Chinensis (L.) Nees}

Local name: Aster

Brief description: A beautiful annual erect and lateral branching herb, sericeous hairy. Inflorescence solitary capitulum peduncle and sessile, incomplete, zygomorphic, hypogynous, calyx modified pappus, florets connate in regular tubular corollas with a cylindric shortly 5-fed limb, $0.6 \mathrm{~cm}$ long basifixed, Carpel 1, style of hermaphrodite florets with under, $0.7 \mathrm{~cm}$ long, stigma bilobate, ovary inferior, one ovule, basal placentation.

Phennology: February to June

Basic chromosome number: $\mathrm{X}=5,8,9$ [4]

Anatomy: Stomata: Upper surface: Anomocytic; Lower surface:Anomocytic. Trichome: Non-glandular; multicellular.

Voucher number: M.R. 35 Motihar, Rajshahi 15-09-07; M.R.39 Sadar, Natore 05-05-05; M.R. 80 Sibgonj, Nawabgonj 11-10-07; M.R. 101 Adamdighi, Bogra 17-11-07; M.R. 117 Raninagar, Naogaon 09-09-07; M.R. 159 Akkelpur, Joypurhat 25-11-07.

\subsection{Calendula Officinalis Linn}

Local name: Calendula

Brief description: A erect annual herb, lateral branched, herbaceous, many angular, hairy. Inflorescence solitary capitulum, epigynous, zygomorphic, pappus dry, ray florets connate in ligulate corolla with a spreading, $3.3 \mathrm{~cm}$ long lamina 2-lobed and entire female flower, hermaphrodite florets connate in regular tubular corolla, stamens 5, syngenesious, carpel 2 , style $0.6 \mathrm{~cm}$ rounded, stigma capitate, ovary inferior one ovule basal placentation.

Phennology: December to February

Basic chromosome number: $\mathrm{X}=7,8,9,12$ [4]
Anatomy: Stomata: Upper surface: Anomocytic; Lower surface:Anomocytic. Trichome: Glandular; multicellular.

Voucher number: M.R. 03 Motihar, Rajshahi 08-04-05; M.R.58 Lalpur, Natore 25-11-07; M.R. 79 Nachole, Nawabgonj 12-10-07; M.R. 104 Adamdighi, Bogra 17-1107.

\subsection{Caesulia Axillaris Roxb}

Local name: Golphuli

Brief description: A glabrous marsh herb; leaves alternate, serrulate. Flower-heads in sessile, axillary, involucrate balls, each sessile on a broad, convex common receptacle, and each 1-flowered. Cypsela included in the laterally compressed bracts; pappus 0 .

Phennology: April to October

Basic chromosome number: $\mathrm{X}=5,7,8$ [4]

Anatomy: Stomata: Upper surface: Anisocytic; Lower surface:Anisocytic. Trichome: Absent.

Voucher number: M.R. 08 Motihar, Rajshahi 15-06-05; M.R.84 Sibgonj, Nawabgonj 16-11-07; M.R. 108 Adamdighi, Bogra 24-12-7.

\subsection{Chrysanthemum Coronarium Linn}

Local name: Chandramollica

Brief description: A annual herb, weak and lateral, branching. Inflorescence capitulum, flower-heads large, corymbose, florets connate in regular tubular corollas, with cylindric or less campanulate, 4-5 lobed limb, $0.6 \mathrm{~cm}$ long, stamens, linear, carpel 1, style of hermaphrodite florets with arms truncate and pennicillate at their tips, ovary superior, 1-loculi and 1 ovule, Style $0.5 \mathrm{~cm}$ long linnet stigma and basal placentation

Phennology: January to April

Basic chromosome number: $\mathrm{X}=9$ [4]

Anatomy: Stomata: Upper surface: Absent; Lower surface:Anisocytic. Trichome: Non-glandular; multicellular.

Voucher number: M.R. 33 Motihar, Rajshahi 25-12-07; M.R.52 Sadar, Natore 22-11-07; M.R. 109 Dupchachia, Bogra 18-11-07.

\subsection{Cosmos Caudatus Kunth}

Local name: Cosmos

Brief description: A showy flowered, tall and annual herb. Inflorescence solitary or loosely corymbose, calyx modified pappus, white, ray-florets 1-seriate, unisexual, petals connate in ligulate corolla with a spreading, $2.9 \mathrm{~cm}$ long, lamina 3-lobed, hermaphrodite florets. Stamens syngenesious; carpel-2, style $0.6 \mathrm{~cm}$ long of hermaphrodite florets with slender arms thickened upwards, ovary inferior, 1-ovule, basal placentation

Phennology: December to March

Basic chromosome number: $\mathrm{X}=12$ [4]

Anatomy: Stomata: Upper surface: Anomocytic; Lower surface:Anomocytic and anisocytic. Trichome: Absent.

Voucher number: M.R. 12 Motihar, Rajshahi 10-10-06; M.R.48 Lalpur, Natore 20-09-06; M.R. 65 Sadar, 
Nawabgonj 05-07-06; M.R. 110 Adamdighi, Bogra 19-1107.

\subsection{Cirsium Arvense (L.) Scop}

Local name: Silkanta

Brief description: An erect, perennial herb, stem not winged cobwebby tomentose above, heads solitary fascicled or corymbose, involucre bracts glabrate or cobwebby outer most short ovate or triangular-ovate ending in a short spine, inner gradually longer with undulate often recurved tips, innermost narrow linear-lanceolate scarious.

Phennology: February to July

Basic chromosome number: $\mathrm{X}=5,8,10,17$ [4]

Anatomy: Stomata: Upper surface: Absent; Lower surface:Anomocytic. Trichome: Non-glandular; multicellular.

Voucher number: M.R. 04 Motihar, Rajshahi 09-04-05; M.R.49 Sadar, Natore 21-09-06; M.R. 85 Sadar, Nawabgonj 17-12-07.

\subsection{Dahlia Variabilis (Willd) Desf}

Local name: Dahlia

Brief description: A long erect annual herb. Inflorescence cymose capitulum, sessile, incomplete, involucre, bracts 3 seriate, free, pappus present, petals of ray florets connate in legislate, , hermaphrodite florets connate in regular tubular corollas with a tubular shortly 5 -fed limb, $1.2 \mathrm{~cm}$ long. Stamens syngenesious; carpel 2, style $0.9 \mathrm{~cm}$ rounded, stigma bilobate and linnet hairy, ovary inferior, 1 locule, 1 ovule, basal placentation

Phennology: January to April

Basic chromosome number: $\mathrm{X}=8,12,18$ [4]

Anatomy: Stomata: Upper surface: Anomocytic; Lower surface:Anomocytic. Trichome: Non-glandular; multicellular.

Voucher number: M.R. 05 Motihar, Rajshahi 10-05-05; M.R.50 Singra, Natore 21-09-06; M.R. 69 Sadar, Nawabgonj 06-07-06; M.R. 91 Adamdighi, Bogra 17-11-06; M.R. 135 Atrai, Naogaon 20-12-07; M.R. 158 Khetlal, Joypurhat 23-12-07.

\subsection{Eclipta Alba (L.) Hassk}

Local name: Kalokeshi

Brief description: Annual, erect or prostrate, branched, herb. Heads 6-8 mm diameter, solitary. Ray-florets ligulate, the ligule small, spreading, scarcely as lon as the bracts, not toothed, white. Disc-florets tubular, the corollas often 4toothed, pappus 0 . Achenes cuneate, compressed and with a narrow wing, covered with warty excrescences.

Phennology: December to May

Basic chromosome number: $\mathrm{X}=4-19$ [4]

Anatomy: Stomata: Upper surface: Anisocytic; Lower surface:Anomocytic . Trichome: Absent.

Voucher number: M.R. 06 Motihar, Rajshahi 11-05-05; M.R.51 Singra, Natore 22-09-06; M.R. 70 Sibgonj, Nawabgonj 07-07-06; M.R. 106 Adamdighi, Bogra 09-1107; M.R. 134 Atrai, Naogaon 20-12-07; M.R. 138 Akkelpur,
Joypurhat 28-07-06.

\subsection{Enhydra Flactuans Lour}

Local name: Hincha, Halencha

Brief description: A pubescent or glabrous herb. Leaves opposite. Inflorescence a sessile head, axillary and terminal; ray florets female, many-seriate, fertile; disk-florets with a 5-fid campanulate limb. Cypsela oblong; enclosed in the rigid pales pappus 0 .

Phennology: January to May

Basic chromosome number: $\mathrm{X}=4-19$ [4]

Anatomy: Stomata: Upper surface: Anomocytic and anisocytic; Lower surface:Anomocytic. Trichome: Absent.

Voucher number: M.R. 16 Boalia, Rajshahi 15-12-06; M.R.43 Lalpur, Natore 23-10-05; M.R. 71 Sadar, Nawabgonj 08-07-06; M.R. 103 Adamdighi, Bogra 20-1107; M.R.113 Raninagar, Naogaon 19-06-06; M.R. 139 Akkelpur, Joypurhat 29-07-06.

\subsection{Eupatorium Odoratum Linn}

Local name: Assamlata

Brief description: A herb, ascending, branching, glabrous. Leaves sub-sessile, opposite. Inflorescence a lax fewheaded corymb, heads pedicillate about 20-flowered. Flowers slaty blue, corollas all equal regular, tubular, anthers appendaged, base obtuse, style-arms long, obtuse. Achenes truncate 5-angled, pappus-hairs uniseriate, many, rigid, scabrid.

Phennology: January to May

Basic chromosome number: $\mathrm{X}=10,17,19$ [4]

Anatomy: Stomata: Upper surface: Anomocytic; Lower surface:Anomocytic and anisocytic. Trichome:Nonglandular; multicellular

Voucher number: M.R. 07 Motihar, Rajshahi 12-05-05; M.R.44 Sadar, Natore 25-10-05; M.R. 73 Sadar, Nawabgonj 10-07-06; M.R. 105 Adamdighi, Bogra 22-1107.

\subsection{Gnaphalium Polycaulon Pers}

Local name: Barakamra

Brief description: An erect, slender, softly cottony. Heads $0.31 \mathrm{~cm}$ diam. in simple or branched leafy spike or racemose clusters, involucre bracts linear oblong acute pale reddish-brown or yellowish, achenes minutely papilose, pappus-hairs not coherent at the base.

Phennology: December to April

Basic chromosome number: $\mathrm{X}=7$ [4]

Anatomy: Stomata: Upper surface: Anomocytic and anisocytic; Lower surface:Anomocytic and anisocytic. Trichome:Non-glandular; multicellular

Voucher number: M.R. 09 Boalia, Rajshahi 16-06-05; M.R.114 Atrai, Naogaon 17-07-06; M.R. 160 Akkelpur, Joypurhat 23-12-07.

\subsection{Gnaphalium Pulvinatum DC}

Local name: Barakamra 
Brief description: An annual herb. Heads minute $0.21 \mathrm{~cm}$ hidden amongst the bases of the crowded spreading terminal leaves and immersed in wool, involucre bracts slender linear recurved green with acute hyaline tips, achenes slender minutely papillose.

Phennology: January to March

Basic chromosome number: $\mathrm{X}=7$ [4]

Anatomy: Stomata: Upper surface: Anomocytic; Lower surface:Anisocytic. Trichome: Non-glandular; multicellular

Voucher number: M.R. 34 Tanore, Rajshahi 13-12-07; M.R.59 Sadar, Natore 05-11-07; M.R. 83 Sadar, Nawabgonj 29-10-07; M.R. 157 Akkelpur, Joypurhat 2312-07.

\subsection{Gnaphalium Indicum Linn}

Local name: Barakamra

Brief description: A softly cottony, stems many from the root ascending leafy, leaves linear-obovate or spathulate apiculate, heads simple or branched leafy spikes or racemose clusters, involucral bracts linear-oblong acute pale raddish-brown or yellowish, achenes minutely papillose, pappus hairs not coherent at the base.

Phennology: January to May

Basic chromosome number: $\mathrm{X}=7$ [4]

Anatomy: Stomata: Upper surface: Anomocytic; Lower surface:Anomocytic and anisocytic. Trichome:Nonglandular; multicellular

Voucher number: M.R. 11 Godagari, Rajshahi 15-06-05; M.R. 88 Adamdighi, Bogra 21-08-06; M.R. 115 Atrai, Naogaon 20-06-06.

\subsection{Grangea Maderaspatana (Linn) Poir}

Local name: Namuti

Brief description: Annual; stems many, hairy with soft white hairs. Heads globose, 6.5-8.5 mm diameter; solitary or 2-nate, on short leaf-opposed peduncles; flowers yellow. Involucral bracts elliptic, obtuse, rigid, densely pubescent. Pappus a short tube with fimbriate mouth. Achenes glandular, $2.5 \mathrm{~cm}$ long including the pappus tube.

Phennology: December to May

Basic chromosome number: $\mathrm{X}=9$ [4]

Anatomy: Stomata: Upper surface: Anomocytic; Lower surface:Anomocytic. Tricome:Non-glandular; multicellular

Voucher number: M.R. 13 Tanore, Rajshahi 17-06-06; M.R.38 Sadar, Natore 26-07-06; M.R. 63 Sibgonj, Nawabgonj 25-08-06; M.R. 89 Dupchachia, Bogra 15-0706; M.R. 133 Raninagar, Naogaon 19-12-07; M.R. 148 Khetlal, Joypurhat 22-09-07.

\subsection{Helianthus Annuus Linn}

Local name: Surjamukhi

Brief description: A tall annual erect herb. Inflorescence solitary capitulum. Ray florets unisexual female 1-seriate, disk florets hermaphrodite all fertile. Stamens syngenesious; 2-carpel, syncarpous style of hermaphrodite florets with arms truncate and pedicillate at their tips, $1.6 \mathrm{~cm}$ long rounded, white, bilobate stigma, linnet hairy, basal placentation.

Phennology: March to September

Basic chromosome number: $\mathrm{X}=17$ [4]

Anatomy: Stomata: Upper surface: Anisocytic; Lower surface:Anomocytic. Trichome: Non-glandular; multicellular

Voucher number: M.R. 14 Tanore, Rajshahi 16-11-05; M.R.40 Lalpur, Natore 20-12-05; M.R. 64 Sadar, Nawabgonj 27-12-06; M.R. 107 Adamdighi, Bogra 19-0705; M.R. 129 Raninagar, Naogaon 14-12-05; M.R. 156 Akkelpur, Joypurhat 11-12-07.

\subsection{Launaea Aspleniifolia (Willd) Hook $f$}

Local name: Tikdana

Brief description: Biennial or perennial, glabrous. Heads $1.3 \mathrm{~cm}$ terminal, paniculate, the peduncles with usually 1 or 2 subulate bracts. Involucral bracts quite glabrous; outer small, inner linear, margins membranous. Achenes $2 \mathrm{~mm}$, columnar, angled and ribbed, ribs rough. Pappus $8 \mathrm{~mm}$, deciduous.

Phennology: December to May

Basic chromosome number: $\mathrm{X}=5,7,8,9$ [4]

Anatomy: Stomata: Upper surface: Anomocytic; Lower surface:Anomocytic and anisocytic. Trichome: Absent.

Voucher number: M.R. 32 Godagari, Rajshahi 07-04-06; M.R.57 Sadar, Natore 03-03-06; M.R. 66 Sadar, Nawabgonj 29-07-06; M.R. 102 Adamdighi, Bogra 17-1207; M.R. 132 Atrai, Naogaon 23-12-07; M.R. 141 Akkelpur, Joypurhat 05-04-06.

\subsection{Lactuca Sativa Linn}

Local name: Lettuce

Brief description: Erect glaucescent annual or biennial, about 0.3-0.9 m high. Capitula 8-15 mm long, on very short pedicels, in a cyme with spreading brances. Flowers about 11, yellow. Achenes striate, dark brown or greyish browns, hispidulous near the top of the body, which terminates in a slender beak of nearly the same length, pappus white.

Phennology: January to May

Basic chromosome number: $X=5,7,8,9$ [4]

Anatomy: Stomata: Upper surface: Anomocytic and anisocytic; Lower surface:Anomocytic. Trichome: Absent.

Voucher number: M.R. 15 Boalia, Rajshahi 16-11-05; M.R.60 Sadar, Natore 12-12-07; M.R. 155 Khetlal, Joypurhat 05-12-07.

\subsection{Mikania Cordata (Burm f) Robinson}

Local name: Assamlata

Brief description: A climbing herb, glabrousor puberulous, heads 4-flowered corymbose terminating lateral branches, achenes glabrous glandular, pappus reddish.

Phennology: December to May

Basic chromosome number: $\mathrm{X}=10,17,19$ [4]

Anatomy: Stomata: Upper surface: Anomocytic; Lower 
surface: Paracytic. Trichome:Non-glandular; multicellular.

Voucher number: M.R. 17 Boalia, Rajshahi 17-11-05; M.R.41 Lalpur, Natore 06-10-05; M.R. 67 Sibgonj, Nawabgonj 30-07-06; M.R. 92 Dupchachia, Bogra 18-0506; M.R. 118 Atrai, Naogaon 24-10-06; M.R. 154 Akkelpur, Joypurhat 24-12-07.

\subsection{Parthenium hysterophorus Linn.}

Local name: Gandi Booti.

Brief description: An erect ephemeral herb known for its vigorous growth. Once stem elongation is initiated, smaller leaves are produced and the plant becomes much branched in its extremities. Pollen is monad, tricolpate and echinate. Shape of pollen in pollar and equatorial view is circular to semi-angular.

Phennology: Throughout the year

Basic chromosome number: $\mathrm{X}=9,18$ [4]

Anatomy: Stomata: Upper surface: Anisocytic; Lower surface:Anomocytic. Trichome: Non-glandular; multicellular.

Voucher number: M.R. 31 Boalia, Rajshahi 07-04-06; M.R.56 Singra, Natore 06-07-06; M.R. 68 Sadar, Nawabgonj 11-12-06; M.R. 100 Adamdighi, Bogra 05-1107; M.R. 131 Atrai, Naogaon 02-11-07; M.R. 153 Akkelpur, Joypurhat 24-12-07.

\subsection{Sonchus Asper (L.) Hill}

Local name: Banpalang

Brief description: Annual, glabrous or sparsely glabrous above; stem branched. Heads crowded in an irregular umbel; flowers yellow. Pappus copious, very slender, white. Achenes $3 \mathrm{~mm}$ long much compressed, elliptic in outline, longitudinally steriate by fine distant ribs, not transversely muriculate.

Phennology: November to June

Basic chromosome number: $X=5,7,8,9$ [4]

Anatomy: Stomata: Upper surface: Absent; Lower surface:Anomocytic. Trichome: Absent.

Voucher number: M.R. 18 Boalia, Rajshahi 18-06-05; M.R.55 Sadar, Natore 25-11-07.

\subsection{Sonchus Arvensis Linn}

Local name: Banpalang

Brief description: A rootstock creeping herb, stem glagrous tall umbellately branched above, leaves runcate pinnatifid spinous-toothed, heads and peduncles glandularhispid, achenes narrow subcompressed with thick regular ribs on each face.

Phennology: November to June

Basic chromosome number: $X=5,7,8,9$ [4]

Anatomy: Stomata: Upper surface: Anisocytic; Lower surface:Anomocytic. Trichome: Absent.

Voucher number: M.R. 19 Boalia, Rajshahi 16-11-05; M.R.42 Sadar, Natore 08-10-06; M.R.82 Sibgonj, Nawabgonj 30-10-07; M.R. 93 Adamdighi, Bogra 19-05-06; M.R. 130 Raninagar, Naogaon 30-10-07; M.R. 142 Khetlal, Joypurhat 10-05-06.

\subsection{Sonchus Winghtianus DC}

Local name: Banpalong

Brief description: A tall perennial. Heads few, $2.4-4.5 \mathrm{~cm}$ across, umbellately corymbose; peduncles glandular- hairy; flowers bright yellow. Involucral bracts clothed with long hairs tipped with greenish yellow glands. Pappus white, silky, scarcely exceeding the involucral bracts. Achenes scarcely compressed.

Phennology: November to June

Basic chromosome number: $\mathrm{X}=5,7,8,9$ [4]

Anatomy: Stomata: Upper surface: Anomocytic; Lower surface:Anomocytic and anisocytic. Trichome: Absent.

Voucher number: M.R. 81 Sibgonj, Nawabgonj 29-10-07; M.R. 152 Akkelpur, Joypurhat 24-12-07.

\subsection{Spilanthes Paniculata Wall ex DC}

Local name: Marhatiga

Brief description: An annual herb, erect or ascending. Heads $0.5-1.4 \mathrm{~cm}$ long, ovoid, solitary or subpanicled; peduncles sometimes reaching or even exceeding $10.3 \mathrm{~cm}$ long. Ray florets and ligules very often absent, the latter when present minute. Pappus 0. Achenes oblong or slightly obovoid, truncate, much compressed.

Phennology: October to January

Basic chromosome number: $\mathrm{X}=7,8,9,13$ [4]

Anatomy: Stomata: Upper surface: Anisocytic; Lower surface:Anomocytic. Trichome: Non-glandular; multicellular.

Voucher number: M.R. 20 Boalia, Rajshahi 17-11-05; M.R.46 Sadar, Natore 14-11-06; M.R. 74 Sadar, Nawabgonj 04-05-07; M.R. 94 Adamdighi, Bogra 02-01-07; M.R. 119 Atrai, Naogaon 07-10-06; M.R. 151 Khetlal, Joypurhat 17-11-06.

\subsection{Synedrella Nodiflora (L.) Gaertn}

Local name: Marhatitiga

Brief description: An erect dichotomously branched herb; stem and branched terete, glabrous. Heads sessile, axillry and terminal: inner involucre bracts linear-lanceolate, shining. Achenes slender, black, spines 2-3 times as long, erect, very stout.

Phennology: September to February

Basic chromosome number: $\mathrm{X}=7,8,9$ [4]

Anatomy: Stomata: Upper surface: Anomocytic; Lower surface:Anomocytic and anisocytic. Trichome: Nonglandular; multicellular.

Voucher number: M.R.30 Tanore, Rajshahi 06-03-06; M.R.47 Sadar, Natore 12-11-06; M.R.75 Sadar, Nawabgonj 11-12-07; M.R. 95 Adamdighi, Bogra 20-05-06; M.R. 120 Atrai, Naogaon 30-05-07; M.R. 143 Akkelpur, Joypurhat 13-05-06.

\subsection{Tagetes Patula Linn}

Local name: Genda

Brief description: A showy-flowered annual erect herb. 
Inflorescence capitulum, solitary. Calyx modified pappus, petals of ray florets connate in a ligule corolla, with flat; of hermaphrodite connate in regular tubular corollas, stamens 5 , anther united, filament free syngenesious; carpel 2, style of hermaphrodite flowers with slender truncate, basal placentation.

Phennology: December to April

Basic chromosome number: $\mathrm{X}=8,12,18$ [4]

Anatomy: Stomata: Upper surface: Anomocytic; Lower surface:Anomocytic. Trichome: Non-glandular; multicellular.

Voucher number: M.R. 21 Boalia, Rajshahi 18-10-06; M.R.53 Sadar, Natore 03-01-07; M.R. 76 Sadar, Nawabgonj 11-12-07; M.R. 96 Adamdighi, Bogra 22-06-06; M.R. 121 Atrai, Naogaon 30-05-07; M.R. 150 Khetlal, Joypurhat 17-11-06.

\subsection{Tridax Procumbens Linn}

Local name: Tridhara

Brief description: A week straggling herb. Leaf simple, opposite. Inflorescence capitulum. Flower: two types, rayflorets and disk-florets. Ray-florets: bracteate, zygomorphic, sessile, incomplete, female, epigynous, basal placentation. Disk-florets: bracteate, sessile, actinomorphic, pentamerous, complete, bisexual, epigynous. Androeceum: stamens 5, gynandrous, syngenesious. Gynoeceum: carpel 2, syncarpous, style 2, stigma 1, ovary inferior, one chambered, basal placentation.

Phennology: Throughout the year

Basic chromosome number: $\mathrm{X}=9$ [4]

Anatomy: Stomata: Upper surface: Anisocytic; Lower surface:Anomocytic. Trichome: Non-glandular; multicellular.

Voucher number: M.R. 29 Boalia, Rajshahi 02-03-06; M.R.54 Sadar, Natore 05-02-07; M.R.77 Sadar, Nawabgonj 11-12-07; M.R.97 Dupchachia, Bogra 30-05-06; M.R. 122 Atrai, Naogaon 30-05-07; M.R. 149 Akkelpur, Joypurhat 15-10-06.

\subsection{Vernonia Patula (Dryand) Merrill}

Local name: Kukshim

Brief description: Annual, erect herb. Heads small, about 20-flowered., $6 \mathrm{~mm}$ diameter, in lax divericate terminal corymbs, with a minute linear bract beneath each head of flowers and with small bracts in the forks of the peduncles; flowers pinkish violet. Pappus white, the exterior row short, about $0.5 \mathrm{~mm}$ long. Achenes $1.25 \mathrm{~mm}$ long, oblong.

Phennology: January to May

Basic chromosome number: $\mathrm{X}=7,8,9$ [4]

Anatomy: Stomata: Upper surface: Anomocytic; Lower surface:Anomocytic. Trichome: Non-glandular; multicellular.

Voucher number: M.R. 22 Boalia, Rajshahi 22-10-06; M.R.161 Sadar, Natore 27-11-07; M.R.78 Sadar, Nawabgonj 11-12-07; M.R. 98 Adamdighi, Bogra 02-06-06; M.R. 123 Atrai, Naogaon 30-05-07; M.R. 144 Akkelpur,
Joypurhat 14-09-06.

\subsection{Vernonia Cinerea (Linn) Less}

Local name: Shiallata

Brief description: A branches woody herb, pubescent. Heads about 20-flowered in loose corymbs, involucral bracts linear-lanceolate tips awned glabrous, pappus white or dirty white outer hairs short.

Phennology: January to May

Basic chromosome number: $\mathrm{X}=7,8,9$ [4]

Anatomy: Stomata: Upper surface: Anomocytic; Lower surface:Anomocytic. Trichome: Non-glandular; multicellular.

Voucher number: M.R. 28 Motihar, Rajshahi 02-03-06.

\subsection{Wedelia Chinensis (Osbeck) Merrill}

Local name: Mohavringaraj

Brief description: A perennial herb. Heads 2-3.3 cm diam.; solitary; Involucral bracts herbaceous, oblong or slightly obovate, hairy, subobtuse, much longer than the disk flowers. Ray-flower ligulate, ligules yellow, 2-3 toothed. Style-arms of female flowers long, acute, recurved. Pappus a toothed membranous cup. Achenes of the ray tapering, slightly pubescent.

Phennology: Throughout the year.

Basic chromosome number: $\mathrm{X}=7,8,9,13$ [4]

Anatomy: Stomata: Upper surface: Anomocytic and anisocytic; Lower surface:Anisocytic and paracytic. Trichome: Non-glandular; multicellular.

Voucher number: M.R. 23 Boalia, Rajshahi 25-10-06; M.R.162 Lalpur, Natore 29-11-07; M.R.166 Sibgonj, Nawabgonj 18-10-07; M.R.99 Adamdighi, Bogra 02-06-06; M.R.126 Raninagar, Naogaon 20-06-07; M.R. 145 Khetlal, Joypurhat 13-11-06.

\subsection{Wedelia Trilobata (L.) A.S. Hitchc}

Local name: Mohavringaraj or creeping daisy

Brief description: Wedelia trilobata is native to the northern part of South America and the West Indies. It is a creeping evergreen perennial that roots at the leaf nodes and spreads widely. The leaves are ovate and usually 3 lobed. The flower is a yellow daisy-like flower that is approximately 1 inch across. Grow in full sun to part shade.

Phennology: Throughout the year.

Basic chromosome number: $\mathrm{X}=7,8,9,13$ [4]

Anatomy: Stomata: Upper surface: Anomocytic; Lower surface:Anisocytic. Trichome: Non-glandular; multicellular. Voucher number: M.R. 27 Boalia, Rajshahi 15-01-08.

\subsection{Xanthium Indicum Linn}

Local name: Banokra, Ghagra

Brief description: Annual, unarmed. Heads in terminal and axillary racemes, the barren heads rather numerous, crowded at the top of the stem, the fertile heads fewer, axillary. Involucre of fertile heads ovoid in fruit, about 1.6 $\mathrm{cm}$ long, with 2 erect mucronate beaks, pubescent, thickly 
clothed with usually hooked prickles, 2-celled, hard and tough. Achenes $1.3 \mathrm{~cm}$ long, oblong-ovoid, compressed, glabrous.

Phennology: December to May

Basic chromosome number: $\mathrm{X}=9,18$ [4]

Anatomy: Stomata: Upper surface: Anisocytic; Lower surface:Anisocytic. Trichome: Non-glandular; unicellular.

Voucher number: M.R. 24 Boalia, Rajshahi 25-10-06; M.R.163 Singra, Natore 08-01-08; M.R.167 Sibgonj, Nawabgonj 15-01-08; M.R.171 Adamdighi, Bogra 19-0108; M.R.127 Atrai, Naogaon 17-12-07; M.R. 147 Akkelpur, Joypurhat 15-12-06.

\subsection{Youngia Japonica (L.) DC}

Local name: Crepis

Brief description: An annual, glabrous or puberulous, radical leaves obovate sinuate-toothed or runcinatepinnatifid flowering stems slender erect nearly naked, heads slender bracteolate peduncles, involucral bracts $6-8$ narrowly oblong glabrous fruiting with a strong basal midrib, achenes narrowly fusiform hardly beaked compressed ribbed 162 qualing or shorter than the white soft pappus.

Phennology: January to May

Basic chromosome number: $\mathrm{X}=5,8,10,17$ [4]

Anatomy: Stomata: Upper surface: Anomocytic; Lower surface:Anomocytic. Trichome: Non-glandular; multicellular.

Voucher number: M.R. 25 Boalia, Rajshahi 03-01-08.

\subsection{Zinnia Peruviana (L.) L}

\section{Local name: Zinnia}

Brief description: An annual erect herb. Inflorescence solitary capitulum. Petals of female ray florets connate in a spreading, entire, ligule, shortly tubular below; of hermaphrodite 5 , connate in regular tubular corollas with a slightly enlarged cylindric, shortly 5 lobed limb, stamens 5, syngenesious; anthers, entire at base, basifixed linear, carpel 1, style with elongated, $0.9 \mathrm{~cm}$ long, white, rounded, ovary inferior, or loculi one ovule, basal placentation.

Phennology: April to August

Basic chromosome number: $\mathrm{X}=7,8,9,12$ [4]

Anatomy: Stomata: Upper surface: Anomocytic and anisocytic; Lower surface:Anomocytic and anisocytic. Trichome: Non-glandular; multicellular.

Voucher number: M.R. 26 Boalia, Rajshahi 03-01-08.

On the basis of morphological, floral, cytological and anatomical characteristics a new system of classification of the family Asteraceae is proposed as follows:

The family is divided into 9 tribes and 16 subtribes.

Tribe 1. Vernonieae: Heads with the flowers all similar and tubular. Anthers cleft at the base. Style-arms subulate, hairy. Leaves usually alternate. Flowers never yellow. Basic chromosome number: $\mathrm{X}=7,8,9$. Stomata anomocytic and trichome non-glandular multicellular. e.g. Vernonia.

Tribe 2. Eupatorieae: Heads with the flowers all similar and tubular. Anthers subentire at the base. Style-arms obtuse, papillose. Leaves opposite or alternate. Corolla rarely orange, never yellow. Pappus usually bristly. Basic chromosome number: $X=10,17,19$. Stomata anomocytic, anisocytic and paracytic. Trichome non-glandular multicellular. e.g. Ageratum, Eupatorium, Mikania.

Tribe 3. Asteroideae: Heads with the flowers all similar, or the outer ligulate. Anthers subentire at the base. Stylearms falttened or plano-convex, all or those of the diskflowers terminated by a cone. Leaves usually alternate. Receptacle almost always naked. Disk florets yellow, ray florets yellow, white or purple.

Subtribe I. Grangeineae: Flowers all yellow, ray 0. Pappus hardly any. Basic chromosome number: $X=9$. Stomata anomocytic and trichome non-glandular multicellular. e.g. Grangea.

Subtribe II. Heterochromeae: Ray-flowers female, ligulate, never yellow. Disk flowers yellow. Pappus hairs long, copious. Basic chromosome number: $X=5,8,9$. Stomata anomocytic and trichome non-glandular multicellular. e.g. Callistephus.

Subtribe III. Conyzeae: Ray-flowers very slender, tubular, or with very short ligules, and disk-flowers yellow or various colour. Pappus copious or scanty. Basic chromosome number: $\mathrm{X}=8,12,18$. Stomata anomocytic and trichome non-glandular multicellular. e.g Dahlia, Tagetes.

Tribe 4. Inuloideae: Heads with the flowers all similar, or the outer ligulate. Anther cells (except Laggera and Phagnalon) tailed. Style arms linear, obtuse, or styles of the sterile fl. undivided. Leaves usually alternate. Disk and ray flowers usually both yellow.

Subtribe I. Plucheineae: Heads androgynous. Involucre bracts dry or herbaceous, rarely subscarious. Receptacle naked. Style-arms of male flower, filiform, not truncate, or style of sterile fl. entire. Basic chromosome number $\mathrm{X}=5$, 7,8 , 9. Stomata anomocytic, anisocytic and trichome nonglandular multicellular. e.g. Blumea.

Subtribe II. Gnaphalieae: Heads androgynous or homogamous. Invol. Bracts scarious, usually hyaline, or the enner radiating. Receptacle usually naked. Style-arms of male flower truncate. Basic chromosome number $\mathrm{X}=7$. Stomata anomocytic, anisocytic and trichome nonglandular multicellular. e.g. Gnaphalium.

Subtribe III. Angiantheae: Heads homogamous, discoid, small, in crowed clusters. Invol. bracts few hyaline. Flower male with truncate style-arms. Basic chromosome number $\mathrm{X}=5$, 7, 8. Stomata anisocytic and trichome absent. e.g. Caesulia.

Tribe 5. Helianthoideae: Heads usually radiate. Receptacle paleaceous. Anther-cells not produced into tails. Style-arms truncate or appendiculate, or style of sterile fl. entire. Achenes 3-4 angled or terete or compressed. Pappus of 2-4 bristles or paleaceous or 0 .

Subtribe I. Ambrosieae: Heads heterogamous or unisexual. Fl. Male sterile with undivided styles; Female flower apetalous. Anthers nearly free, with inflexed 
appendages. Basic chromosome number $X=9$, 18. Stomata anisocytic and trichome non-glandular unicellular. e. g. Xanthium.

Subtribe II. Verbesineae: Heads hetero or homogamous. Fl. Male all fertile. Achenes of the disk angled, subterete, or laterally compressed, crowned with 2-3 bristles or scales or naked. Leaves usually opposite. Basic chromosome number $\mathrm{X}=4-19$. Stomata anomocytic, anisocytic and trichome absent. e.g. Enhydra, Eclipta.

Subtribe III. Wedeloideae: Heads hetero or homogamous. Fl. Male all fertile. Leaves usually opposite. Flower colour yellow. Basic chromosome number $\mathrm{X}=7,8,9$, 13. Stomata anomocytic, anisocytic and paracytic. Trichome nonglandular multicellular. e.g. Wedelia, Spilanthes.

Subtribe IV. Coreopsideae: Heads hetero or homogamous. Fl. Male all fertile or neuter. Achenes dorsally compressed, 2-4 awned or naked. Basic chromosome number $\mathrm{X}=7,8,9$. Stomata anomocytic and trichome non-glandular multicellular. e.g. Synedrella.

Subtribe V. Galinsogene: Heds hetero or homogamous. Fl. Male all fertile. Achenes with short bristle like palea. Basic chromosome number $X=9$. Stomata anomocytic, anisocytic and trichome non-glandular multicellular. e. g. Tridax.

Subtribe VI. Heliantheae: Heads usually radiate and large. Receptacle with a chafflike bracts (scale) subtending each disc flower. Basic chromosome number $X=17$. Stomata anomocytic, anisocytic and trichome non-glandular multicellular. e. g. Helianthus.

Tribe 6. Anthemideae: Heads heterogamous, rayed or disciform or rayless and homogamous. Invol. bracts 2seriate, dry or with scarious tips. Receptacle naked or paleaceous. Anther-cells not produced into tails. Style-arms truncate. Pappus 0 or a crown of short paleae. Leaves usually alternate. Disk flowers yellow, ray flowers variously coloured. Stomata anomocytic, anisocytic and trichome non-glandular multicellular.

Subtribe I. Chrysanthoideae: Leaves usually alternate. Disk flowers yellow, ray flowers variously coloured. Basic chromosome number $\mathrm{X}=9$. Stomata anisocytic and trichome non-glandular multicellular. e. g. Chrysanthemum.

Subtribe II. Leaves usually alternate. Disk flowers yellow, ray flowers variously coloured. Basic chromosome number $\mathrm{X}=12$. Stomata anomocytic, anisocytic and trichome absent. e. g. Cosmos.

Tribe 7. Caledulaceae: Heads rayed. Invol. bracts 1-2seriate, narrow, subequal. Receptacle naked. Anther cells mucronate. Style-arms of male fl. truncate or in sterile fl. undivided. Achenes usually large and thick and deformed, without pappus. Basic chromosome number $\mathrm{X}=7,8,9,12$. Stomata anomocytic, anisocytic and trichome nonglandular or glandular multicellular. e. g. Calendula, Zinnia.

Tribe 8. Perthenideae: An erect rphemeral herb known for its vigorous growth. It is light green with branching stems, finely lobed leaves and grows up to 1.5 meters, occasionally reaching $2 \mathrm{~m}$ in deep rich soils. Young plants form a basal rosette of strongly dissected leaves that are up to $30 \mathrm{~cm}$ in length. Once stem elongation is initiated, smaller leaves are produced and the plant becomes muchbranched in its extremities. Basic chromosome number $\mathrm{X}=9$, 18. Stomata anomocytic and trichome non-glandular multicellular e. g. Parthenium.

Tribe 9. Cichoriaceae: Annual or perennial herbs; heads sessile or peduncled, solitary fascicled, yellow or red; homogamous; involucre bracts, inner 1-seriate; receptacle flat, naked or subfimbrillate. Achenes truncate or narrowed or beaked. Pappus short, of scales or bristles or hairy or 0 .

Subtribe I. Lactusoideae: Annual or perennial herbs; heads sessile or peduncled, solitary fascicled, yellow or red. Basic chromosome number $\mathrm{X}=5,7,8$, 9. Stomata anomocytic, anisocytic and trichome absent. e. g. Lactuca, Sonchus, Launaea.

Subtribe II. Crepisoideae: Annual or perennial herbs; heads sessile or peduncled, solitary fascicled, yellow or red. Basic chromosome number $\mathrm{X}=5,8,10,17$. Stomata anomocytic and trichome non-glandular multicellular. e. g. Youngia, Circium.

\section{Conclusion}

Systematic investigation on the family Asteraceae (Compositae) growing throughout in the northern region of Bangladesh was carried out. A total of 36 species under 29 genera of the family Asteraceae were collected and identified. Finally, a new system of classification of the family Asteraceae has been proposed on the basis of morphological, anatomical, cytological and floral characters.

\section{Acknowledgements}

The author is grateful to the Ministry of Science, Information and Communication Technology, Government People's Republic of Bangladesh for financial support to complete this research work.

\section{References}

[1] Ahmed Z U, Begum Z N T, Hassan M A, Khondker M, Kabir S M H, Ahmad M, Ahmed A T A, Rahman A K A and Haque E U(Eds). Encyclopedia of Flora and Fauna of Bangladesh. Angiosperms; Dicotyledons. Asiat. Soc. Bangladesh, Dhaka, Vol 6, 2008; pp.263-359.

[2] Bhattacharyya B and Johri BM. Flowering Plants Taxonomy and Phylogeny. Prokas Publishers, Calcutta, India., 1998.

[3] Cronquist A. The Evolution and Classification of Flowering Plants. Houghton Miftlin, Boston. U.S.A., 1968.

[4] Darlington CD and Wylie AP. Chromosome Atlas of Flowering Plants. George Allen and Unwin Ltd. Ruskin House Museum Street, London, U.K., 1950; pp.245-271.

[5] Heywood VH. Flowering Plants of the World. Oxford University Press, New York, U.S.A., 1979. 
[6] Hooker JD. Flora of British India. L. Reeve and Co. Ltd. London, U.K., Vol 1-7, 1961; pp.220-419.

[7] Hossain ABME and Hassan MA. Wedelia trilobata ( L. ) A. S. Hitchc. (Asteraceae)- A New record for Bangladesh. Bangladesh Journal of Plant Taxon. 2005; 12 (1): 63-65.

[8] Huq AM. Name Changes in Bangladesh Angiosperms. Bangladesh National Herbarium, BARC, Dhaka, Bangladesh., 1986; pp.1-289.

[9] Huq AM. Plant Names of Bangladesh. Bangladesh National Herbarium, BARC, Dhaka, Bangladesh., 1986; pp.1-104.

[10] Judd WS, Campbell CS, Kellogg E and Stevens PF. Plant Systematics :A Phylogenetic Approach. Sinauer, Sunderland, Massachusetts, U.S.A., 1999.

[11] Khatun R. Plant Taxonomy. Eureka Book Agency, New Market, Rajshahi, Bangladesh., 2002; pp.384-393.

[12] Kirtikar KR and Basu BD. Indian Medicinal Plants. Lalit Mohan Basu, Allahabad, Jayyed Press, New Delhi, India. Vol 1-4, 1987; pp.1313-1449.

[13] Lawrence GHM. Taxonomy of Vascular Plants. Oxford and IBM Publishing Co., Rakes Press, New Delhi, India., 1973.
[14] Naik VN. Taxonomy of Angiosperms. Tata McGraw-Hill Publishing Company Limited, New Delhi. India., 2003; pp.74-160.

[15] Pasha MK and Zaman MB. Name Changes in Plants of Bangladesh. Chittagong University Studies, Part-II, Science Vol. 12(1), 1988; pp.107-112.

[16] Pasha MK and Uddin SB. Dictionary of Plant Names of Bangladesh. Janokalyan Prokashani, Chittagong-4000, Bangladesh., 2013; pp.1-434.

[17] Prain D. Bengal Plants. Botanical Survey of India. Calcutta, India., Vol 1-2, 1963; pp.580-630.

[18] Rahman A H M M, Islam A K M R and Rahman M M. The Family Asteraceae of Rajshahi Division, Bangladesh, VDM Verlag Dr. Muller Publishing House Ltd., Germany., 2011; pp.1-176.

[19] Sambamurty AVSS. Taxonomy of Angiosperms. I.K. International Pvt. Ltd. New Delhi. India., 2005; pp.408-417.

[20] Sharma OP. Plant Taxonomy. Tata Mc Grow Hill Publishing Co. Ltd. New Delhi, India., 2004; pp.312-318. 